\title{
CHARACTERISTIC MULTIPLIERS FOR SOME PERIODIC DIFFERENTIAL EQUATIONS
}

\author{
T. G. PROCTOR ${ }^{1}$
}

1. Introduction. Let $P(t)$ be a $2 \times 2$ matrix with elements which are continuous real valued functions of period $T$ and consider the differential equation

$$
\dot{x}=P(t) x
$$

where $x$ is a vector with two components. It is well known [7] that there are numbers $\lambda_{1}$ and $\lambda_{2}$, called characteristic multipliers, and corresponding solutions $x_{1}(t), x_{2}(t)$ of $(1)$, called normal solutions, which satisfy for $i=1,2$,

$x_{i}(t+T)=\lambda_{i} x_{i}(t), \quad-\infty<t<\infty, \quad \lambda_{1} \lambda_{2}=\exp \int_{0}^{T} \operatorname{trace} P(t) d t$.

If $\lambda_{1} \neq \lambda_{2}$, and in some cases when $\lambda_{1}=\lambda_{2}$, any such normal solutions $x_{1}(t), x_{2}(t)$ are independent. In this case a knowledge of the characteristic multipliers and the values of $x_{1}(t), x_{2}(t)$ for $0 \leqq t \leqq T$ gives information for every solution of (1) for all $t$. It is clear that corresponding statements can be made for the second order equation

$$
d^{2} y / d t^{2}+p(t)(d y / d t)+q(t) y=0,
$$

with continuous periodic coefficient functions $p(t), q(t)$ since this results from the case

$$
x=\left|\begin{array}{c}
y \\
d y / d t
\end{array}\right|, \quad P(t)=\left|\begin{array}{cc}
0 & 1 \\
-q(t) & -p(t)
\end{array}\right| .
$$

Calculation of the characteristic multipliers is not routine since in general one does not know even one nontrivial solution of (1). However it is possible to obtain convergent series representations for the solutions and thus calculate approximate values for the multipliers [2], [8].

An alternative procedure for obtaining the characteristic multipliers and the corresponding normal solutions for (1) is possible when1968.

Presented to the Society, November 9, 1968; received by the editors August 8,

1 This work was supported by the National Aeronautics and Space Administration under Research Grant NGR 41-001-016. 
ever an associated Riccati differential equation has a periodic solution. If we make the change of coordinates $x^{1}=z^{1}+y(t) z^{2}, x^{2}=z^{2}$ in (1) where $y(t)$ is a solution of the Riccati equation

$$
\begin{gathered}
d y / d t=a(t)+b(t) y+c(t) y^{2}, \\
a(t)=p_{12}(t), \quad b(t)=p_{11}(t)-p_{22}(t), \quad c(t)=-p_{21}(t),
\end{gathered}
$$

the differential equation in $z$ can be integrated. This gives

THEOREM 1. (a) If $x(t)=$ column $\left(x^{1}(t), x^{2}(t)\right)$ is a solution of (1) then $y(t)=x^{1}(t) / x^{2}(t)$ is a solution of (2) on any interval on which $x^{2}(t)$ does not vanish.

(b) If $y(t)$ is a solution of (2) on an interval I containing the number $k$ then

$$
\begin{aligned}
& x^{1}(t)=y(t) \exp \int_{k}^{t}\left[p_{21}(s) y(s)+p_{22}(s)\right] d s, \\
& x^{2}(t)=\exp \int_{k}^{t}\left[p_{21}(s) y(s)+p_{22}(s)\right] d s
\end{aligned}
$$

is a solution of (1) on $I$.

(c) If $y(t)$ is a solution of (2) with period $n T$ and $f$ is the mean value of $p_{21}(t) y(t)+p_{22}(t)$ over the period $n T$, where $n$ is a positive integer, then $e^{n T f}$ is a characteristic multiplier for (1) for the period $n T$ and (3) is a normal solution of (1) corresponding to this multiplier.

In $\$ \$ 2$ and 3 of this paper we will prove two theorems with rather restrictive hypotheses which give the existence of a periodic solution of (2). The theorem in $\$ 2$ can be viewed as a special case of the theorem in $\$ 3$. We also mention other known techniques for constructing periodic solutions to the Riccati differential equation.

Similar analysis for the differential equation (1) where $P(t)$ is $n \times n$ and $x$ is an $n$ vector leads to the study of a matrix Riccati differential equation and the analysis is more difficult. The technique of using a Riccati differential equation has been used by Gel'man [3] and Adrianova [1] for the case of quasi-periodic coefficients $p_{i j}(t)$.

2. $b(t)$ has nonzero mean value. Let $H$ be the set of all continuous real valued functions with period $T$, let $b \in H$, let $B(t)=\int_{0}^{t} b(s) d s$ and in this section we will assume $B(T) \neq 0$. Also suppose $u, l \in H$ and satisfy $l(t) \leqq u(t)$ for all $t$ and let $K$ be the subset of $H$ consisting of functions $f$ where $l(t) \leqq f(t) \leqq u(t)$. Further assume $q(t, z)$ is a continuous real valued function defined for $l(t) \leqq z \leqq u(t)$ such that for any fixed $z, q \in H$ and define $\jmath: K \rightarrow H$ by 


$$
J h(t)=\frac{1}{e^{-B(T)}-1} \int_{t}^{t+T} q(s, h(s)) \exp \int_{s}^{t} b(v) d v d s .
$$

ThEOREM 2. If (1) $J u(t) \leqq u(t), l(t) \leqq \Im l(t)$ for all $t$ and $(2) e^{-B(T)}-1$ $>0$ and $z \leqq w$ implies $q(t, z) \leqq q(t, w)$ or $(3) e^{-B(T)}-1<0$ and $z \leqq w$ implies $q(t, z) \geqq q(t, w)$, then the sequences $\left\{J^{m} u(t)\right\}_{m=1}^{\infty}$ and $\left\{J^{m} l(t)\right\}_{m=1}^{\infty}$ converge uniformly to periodic solutions of

$$
d z / d t=b(t) z+q(t, z) .
$$

Proof. The hypotheses (1) and (2) or (1) and (3) imply that if $h$, $k \in K$ and $h(t) \leqq k(t)$ for all $t$ then $J h(t) \leqq J k(t)$ for all $t$. Thus the sequences $\left\{J^{m} l(t)\right\}_{m=0}^{\infty}$ and $\left\{J^{m} u(t)\right\}_{m=0}^{\infty}$ are nondecreasing and nonincreasing respectively, uniformly bounded and equicontinuous.

REMARKS. If $z(t)$ is a periodic solution of (4) where $q(t, z)=c(t)$ $(w(t)+z)^{2}$,

$$
w(t)=\frac{1}{e^{-B(T)}-1} \int_{t}^{t+T} a(s) \exp \int_{o}^{t} b(v) d v d s
$$

we have that $y(t)=w(t)+z(t)$ is a periodic solution of (2). If $y(t)$ is a periodic solution of (4) where $q(t, y)=a(t)+c(t) y^{2}$ then $y(t)$ is a periodic solution of (2).

EXAMPLE. If we regard the right member of $\dot{y}=(y-1) \times(y-2)$ as having period $2 \pi$ the corresponding transformation $J$ is given by

$$
J h(t)=\frac{e^{-3 t}}{e^{6 \pi}-1} \int_{t}^{t+2 \pi} e^{3 s}\left[h^{2}(s)+2\right] d s .
$$

We note $u(t)=5 / 4, l(t)=0$ satisfy the conditions of the theorem and $J^{m} l(t), J^{m} u(t)$ converge to 1 as $m \rightarrow \infty$.

Several theorems on periodic solutions of (2) are given in Hale [6, pp. 28-31] which result from the contraction mapping theorem and successive approximations. Using Theorem 5.1 [6] a periodic solution exists if (1) $|c(t)|$ is small enough $\left(q(t, z)=c(t)(w(t)+z)^{2}\right.$, $y=w(t)+z(t))$ or (2) if $|a(t)|$ is small enough $\left(q(t, y)=a(t)+c(t) y^{2}\right)$. We state without proof an obvious modification (for the scalar case) of [6, Theorem 5.2] because of its relation to (1). Let $h \in H$ and have mean value zero. Let $r$ and $\sigma$ be positive numbers and let $q(t, y, \mu)$ be a continuous real valued function for all $t,-r \leqq y \leqq r$ and $|\mu| \leqq \sigma$. Further let $q$ be periodic for fixed $(y, \mu)$ and satisfy for $|y|,|z| \leqq \rho$, $|\mu| \leqq \sigma|q(t, y, \mu)-q(t, z, \mu)| \leqq \eta(\mu, \rho)|y-z|, q(t, 0,0)=0$, where $\eta(\mu, \rho)$ is continuous and nondecreasing in $\mu$ and in $\rho$ for $|\mu|<\sigma$, $0 \leqq \rho \leqq r$. 
THEOREM 3. There is a $\delta, 0<\delta<\sigma$, so that for $|\mu|<\delta$

$$
d y / d t=\mu[b(t) y+q(t, y, \mu)+h(t)]
$$

has a periodic solution which is the limit of successive approximations.

Corollary. Let $p(t, \mu), c(t, \mu)$ be continuous real valued functions defined on $R \times[0, \sigma]$ which satisfy $p(t, 0)=c(t, 0)=0, p, c \in H$ for fixed $\mu$, let $q, r, h \in H, h$ with zero mean value, $r$ with nonzero mean value and let

$$
P(t)=P(t, \mu)=\mu\left(\begin{array}{cc}
q(t) & p(t, \mu)+h(t) \\
-c(t, \mu) & q(t)-r(t)
\end{array}\right) .
$$

Then for $\mu$ sufficiently small there is a periodic solution $y(t)$ of (2) and $e^{T f}$ is a characteristic multiplier for (1) where $f$ is the mean value of $p_{11}(t) y(t)+p_{22}(t)$.

By replacing $t$ by $t / \mu$ we see that an alternative statement for this corollary is that there is a number $\lambda$ and a solution of

$$
\dot{x}=\left(\begin{array}{cc}
q(t / \mu) & p(t / \mu, \mu)+h(t / \mu) \\
-c(t / \mu, \mu) & q(t / \mu)-r(t / \mu)
\end{array}\right) x
$$

satisfying $x(t+\mu T)=\lambda x(t)$ when $\mu$ is small enough.

Wasow [9] and Golomb [4] have developed a recursive scheme for constructing periodic solutions for the quasilinear differential equation $y=b(t) y+q(t, y, \mu)$ when $b(t)$ has nonzero mean value and $\mu$ is small. The resulting theorems provide another technique for obtaining periodic solutions of (2) and thus obtaining characteristic multipliers for (1). Golomb [5] also shows that in certain cases this recursive scheme leads directly to a calculation of the characteristic multipliers and corresponding normal solutions.

3. General case. Let $H^{\prime}$ be any closed subspace of $H$ in the uniform topology and for any $f \in H$ define $M f=(1 / T) \int_{0}^{T} f(t) d t$. Let $l, u$ be in $H^{\prime}$ with $l(t) \leqq u(t)$ for all $t$ and let $K$ be all functions in $H^{\prime}$ lying between $l$ and $u$. Further suppose $q(t, y, \mu)$ is defined and continuous for $l(t) \leqq y \leqq u(t)|\mu| \leqq \sigma$ for some $\sigma>0$ and has period $T$ for fixed $(y, \mu)$. For any number a satisfying $l(0) \leqq a \leqq u(0)$ and $|\mu| \leqq \sigma$ define $J_{a, \mu}: K \rightarrow H$ by

$$
\Im_{a, \mu} f(t)=a+\int_{0}^{t}[q(s, f(s), \mu)-M\{q(t, f(t), \mu)\}] d s .
$$

THEOREM 4. For fixed $(a, \mu)$ if $\Im_{a}(K) \subset H^{\prime}, J_{a, \mu} u(t) \leqq u(t), l(t)$ $\leqq J_{a, \mu} l(t)$ for all $t$ and if $y, z \in K, y(t) \leqq z(t)$ implies $\mathfrak{J}_{a, \mu} y(t) \leqq J_{a, \mu} z(t)$ for 
all $t$ then the sequences $\left\{J_{a, \mu}^{m} l(t)\right\}_{m=0}^{\infty},\left\{J_{a, \mu}^{m} u(t)\right\}_{m=0}$ converge uniformly to functions $l^{*}(t, a, \mu), \mu^{*}(t, a, \mu)$ satisfying

$$
\frac{d y}{d t}(t, a, \mu)=q(t, y(t, a, \mu), \mu)-M\{q(t, y(t, a, \mu), \mu)\} .
$$

The proof is identical to that of Theorem 2 .

CoRollary. If $l^{*}(t, a, \mu)$ exists and $M\left\{q\left(t, l^{*}(t, a, \mu), \mu\right)\right\}=0$ then $l^{*}$ is a periodic solution of

$$
d y / d t=q(t, y, \mu) .
$$

An analogous statement holds for $u^{*}(t, a, \mu)$.

As an example of this theorem and corollary consider $q(t, y, \mu)=y$ $\sin t, a=1$, and $H^{\prime}$ the set of all even functions in $H, l(t) \equiv 1$ and $u(t)=\exp (1-\cos t)$.

Hale [6, pp. 38-44] gives several theorems for periodic solutions of $d y / d t=b(t) y+q(t, y, \mu)$ in the case where $b(t)$ has zero mean value and $q$ is small for small $\mu$. These theorems again use successive approximation and the contraction mapping theorem. In the application of these theorems to the differential equations the symmetry properties of $q(t, y, \mu)$ play an important role in the analysis to determine if the bifurcation equations $M q(t, y(t))=0$ have a solution. One of the simplest cases arises when $b(t)=0$ and $q(t, y, \mu)$ is odd in $t$. In this particular case the recursive method developed by Wasow and Golomb also gives a convergent series expansion for the solution for small $\mu$.

4. Final remarks. Note that if $T$ is the least period of $P(t)$ in (1) then the coefficient functions $a, b, c$ in (2) have least period 0 or $T / n$ for some positive integer $n$. By applying the construction procedures used in Theorems 2, 3, or 4, we construct solutions of (2) with period $T / n$ or 0 . Then by replacing $n T$ by $T$ in Theorem 1 , we obtain a characteristic multiplier for the period $T$.

For a given matrix $Q(t)$ we notice that all matrices $P(t)=Q(t)+K(t)$ have the same associated Ricatti differential equation (2) if $K(t)$ has the form

$$
K(t)=\left(\begin{array}{cc}
p(t) & 0 \\
0 & p(t)
\end{array}\right) .
$$

If for some such $K(t)$ equation (1) can be solved, Theorem 1 may be used to solve equation (2). This in turn furnishes a method to solve $\dot{x}=Q(t) x$. This remark applies even if none of the functions in $Q(t)$ 
and $K(t)$ are periodic. However in the case of periodic $P(t)$ suppose $\dot{x}=P(t) x$ has a normal solution, column $\left(x^{1}(t), x^{2}(t)\right)$ where $x^{2}(t)$ does not vanish. If $\lambda$ is the corresponding characteristic multiplier, $y(t)$ $=x^{1}(t) / x^{2}(t)$ and $g=M p(t)$, then $\lambda e^{T o}$ is a characteristic multiplier for $\dot{x}=Q(t) x$ and a corresponding normal solution is given by (3) where $p_{22}$ is replaced by $p_{22}-p$.

Since the proof of Theorem $1 \mathrm{a}$ and $\mathrm{b}$ does not require the periodicity of $P(t)$, theorems which imply the existence of bounded or almost periodic solutions of (2) or the asymptotic form of certain solutions of (2) also give information concerning the solutions of (1).

\section{REFERENCES}

1. L. Ya. Adrianova, The reducibility of systems of $n$ linear differential equations with quasi-periodic coefficients, Vestnik Leningrad Univ. 17 (1962), no. 7, 14-26. (Russian)

2. N. P. Erugin, Linear systems of ordinary differential equations, Academic Press, New York, 1966.

3. A. E. Gel'man, On the reducibility of a class of systems of differential equations with quasiperiodic coefficients, Dokl. Akad. Nauk SSSR 116 (1957), 535-537.

4. M. Golomb, Solution of certain nonautonomous differential systems by series of exponential functions, Illinois J. Math. 2 (1959), 45-65.

5. - Expansion and boundedness theorems for solutions of linear differential systems with periodic or almost periodic coefficients, Arch. Rational Mech. Anal. 2 (1958), 283-308.

6. J. K. Hale, Oscillations in nonlinear systems, McGraw-Hill, New York, 1963.

7. P. Hartman, Ordinary differential equations, Wiley, New York, 1964.

8. I. G. Malkin, Theory of stability of motion, AEC Translation 3352, U. S. Department of Commerce, Washington, 1952.

9. W. Wasow, Solution of certain nonlinear differential equations by series of exponential functions, Illinois J. Math. 3 (1959), 45-65.

\section{Clemson University}

